\title{
A Review of Optical Imagery and Airborne LiDAR Data Registration Methods
}

\author{
Rakesh Kumar Mishra* and Yun Zhang
}

Department of Geodesy and Geomatics Engineering, University of New Brunswick, Canada

\begin{abstract}
Representing a scene completely from remote sensing data requires both spectral and 3-D-surface information. Integration of spectral information from optical images and 3-D-surface information from LiDAR is important in a number of remote sensing applications such as feature extraction, image classification, image analysis, building extraction, 3-D city modelling, canopy modelling etc. Therefore, numerous methods have been developed in the last decade to align both data sets into a common reference frame to effectively utilize their complementary characteristics. However, due to the significantly different characteristics between optical image and LiDAR data, there are a number of technical challenges in the alignment of both data sets. Different research papers introduced different strategy or methodology to overcome the challenges, reaching different alignment/registration results. This paper presents a review of classical and up to date optical-LiDAR registration methods with the emphasis on control point detection and matching. The aim of this paper is to provide readers with an overview of existing methods, identify their advantages and limitations, and give readers the overall information on what will be useful for researchers and practitioners to realistically select proper method for their application.
\end{abstract}

Keywords: Registration, optical image, LiDAR, control point, feature detection, feature matching.

\section{INTRODUCTION}

The technological advancement in computer, sensor technology, data processing and communication led to the launch of the new generation of High Resolution (HR) commercial Earth imaging satellites. The HR satellite image has the advantage of high user interpretability, rich information content, high image clarity, and integrity,which opens the door for many new applications. The recently emerged technique of airborne altimetricLiDAR (Light detection and ranging) provides accurate 3-D surface information whichhelps in deciphering 3-D geometric informationof ground features. However, sudden elevation changes along the surface are not clearly visible in the LiDAR data due to insufficient points. The LiDAR data provides high density surface information in homogenous areas and low density surface elsewhere (i.e. object space break-lines). On the other hand, an optical image provides high quality details along object boundaries with height variations [1]. Optical images and LiDAR data have unique characteristics that make them preferable in certain applications. The disadvantage of one technology is contrasted by an opposite strength in the other. Hence, integrating of data from these two systems would lead to higher quality surface information [2]. Accurate registration of optical images and LiDAR data is important in a number of remote sensing applications such as:

*Address correspondence to this author at the Department of Geodesy and Geomatics Engineering, University of New Brunswick, Canada;

Tel: +1-506-452-6134; Fax: +1-506-453-4943;

E-mails: rakesh.mishra@unb.ca, rkmishramca@gmail.com a. Building extraction: In the past, many efforts have been made to automatically extract buildings from optical images or LiDAR derived DSM. Building extraction using either optical image or LiDAR data has limitations due to the fact that both sensors have some limitations.Integration of these two data sets can overcome the limitations of optical and LiDAR sensors as both sensors have complementary characteristics. Many recent studies to extract buildings, use both optical image and LiDAR data together to improve the accuracy of the building extraction.Rottensteiner and Jansa [3] have shown that the LiDAR data integration with optical imagery is helpful in building extraction. Integration of optical image with LiDAR data facilitates utilizingthe characteristics of both data sets in building extraction. However, the prerequisite of using both data sets together isaccurate alignment of both data sets in a common reference frame.

b. Image classification: At present, the image classificationalgorithms mainly use either spectral or contextual informationof optical images for landcover classifications. The object-oriented image classification method has been found better over the pixel-based image classification methodsparticularly for HR optical images. However, there is still enough scope in improving the image classification so the results can be used on operational basis. Integration of optical images and LiDAR data can greatly improve the classification accuracy by sharing redundant and complementary information of both data sets [4]. A number of research works have been done to utilize LiDAR data to improve the classification of optical 
images $[4,5,6,7]$. However, to use LiDAR data in image classification, the LiDAR data should be perfectly registered with the corresponding optical image.

c. 3-D City Modelling:3-D city modelling is useful in understanding a scene of interest [8]. This has gained popularity in games and many commercial applications. 3-D city modelling has many valuable applications such as urban planning and simulation, virtual reality, interpretation, and real-time emergency response. 3-D models are constructed by texture mapping of optical images onto 3D geometry models. These models have traditionally been constructed manually. The LiDAR technology has made the acquisition of high resolution elevation data more efficient and cost effective. Brenner [9] reviewed many building reconstruction methods and concluded that combining optical imageswith LiDARdata is the better way to increase automation and obtain accurate results.However, using LiDAR data in 3-D city modelling needs optical images accurately registered with corresponding LiDAR data.

d. Canopy modelling:Many research works have utilized the spectral characteristics of optical images to detect forest and tree crowns. However, optical images are not good enough to model canopy structure as it does not capture forest structure directly. As LiDAR data provides very accurate and dense horizontal and vertical information, the canopy height can directly be retrieved from LiDAR data. Chen et al. [10] have shown that the use of LiDARdata with optical images is useful in canopy modelling. The precise canopy modelling requires optical images accurately registered with corresponding LiDARdata.

However, due to the distinguished differences between optical image and LiDAR data, the registration remains challenging.

\section{DIFFICULTIES IN OPTICAL IMAGE AND LIDAR DATA REGISTRATION}

A complete scene description from remote sensing data requires both spectral and 3-D surface information. However, the complementary information of optical images and LiDAR data can be fully utilized only after precise registration of both data sets. Therefore, registration of optical images and LiDAR data models relative to a common reference frame $[11,12]$ is highly beneficial for many remote sensing applications. The optical image can be aligned with the corresponding LiDAR datamanually or automatically through an image registration process using control points. The manual process is performed by selecting control point pairs from both data sets. This process is very time consuming and is prone to human bias given a large area. Therefore, automated registration process is highly desirable to reduce processing time.An optical image and corresponding LiDAR data are shown in Fig. (1). In Fig. (1), the potential control points, building corners are highlighted with yellow circles. There are various important issues which make optical image and LiDAR data registration a quite difficult task [13]. These are given as follows: a. Control-Point Detection: To register a pair of images with each other, a set of conjugate control-points must be detected from both images to compute the registration parameters. However, there are substantial differences in characteristics of optical images and LiDAR data. Therefore, it is very difficult to determine the same points of interest in both data sets.

b. Difference in characteristics of optical image and LiDAR intensity image s:There are substantial differences betweenan optical image and a LiDAR intensity image due to their very different processes of intensity recording. This makes it difficult to perform a direct similarity comparison between a LiDAR intensity image and an optical image. However, the intensity image from LiDARsensor can be used to improve the registration accuracy. Further, the elevation image (DSM) obtained by LiDAR data has also very different characteristics than the optical image.

c. Different Structure:As the optical and LiDARsensorscapture data in entirely different environments, the structural characteristics recorded by optical image may not be present in LiDAR data or vice-versa. This makes it difficult to perform comparisons of structures appearing in both data sets such as edges and shapes.

d. Relief displacement: In the HR optical images relief displacement is highly apparent. Relief displacement shifts the position of an object's image caused by the relief of the object. There is no relief displacement in LiDAR data. Therefore, shift of objects (controlpoints) in optical images makes it difficult to associate control-points of optical images with control-points in LiDAR data.

\section{OPTICAL IMAGE AND LIDAR DATA REGISTRA- TION METHODOLOGY}

The optical images and LiDAR data registration methods consist of following three steps:

(1). Feature Extraction : Salient and distinctive features (homogenous regions, edges, object boundary, lines, line intersection, corners, etc.) are manually or automatically detected from optical images and LiDAR data. These features can be represented by their lines, corners, center of gravity, etc.which are called control points.

(2). Feature matching: This step determines correspondence between the control points detected in optical images and LiDAR data. Many similarity measures and feature descriptors are used for matching the control points.

(3). Transformation model: Image transformation parameters are computed using correspondence between the matched control points from optical images and LiDAR data. 

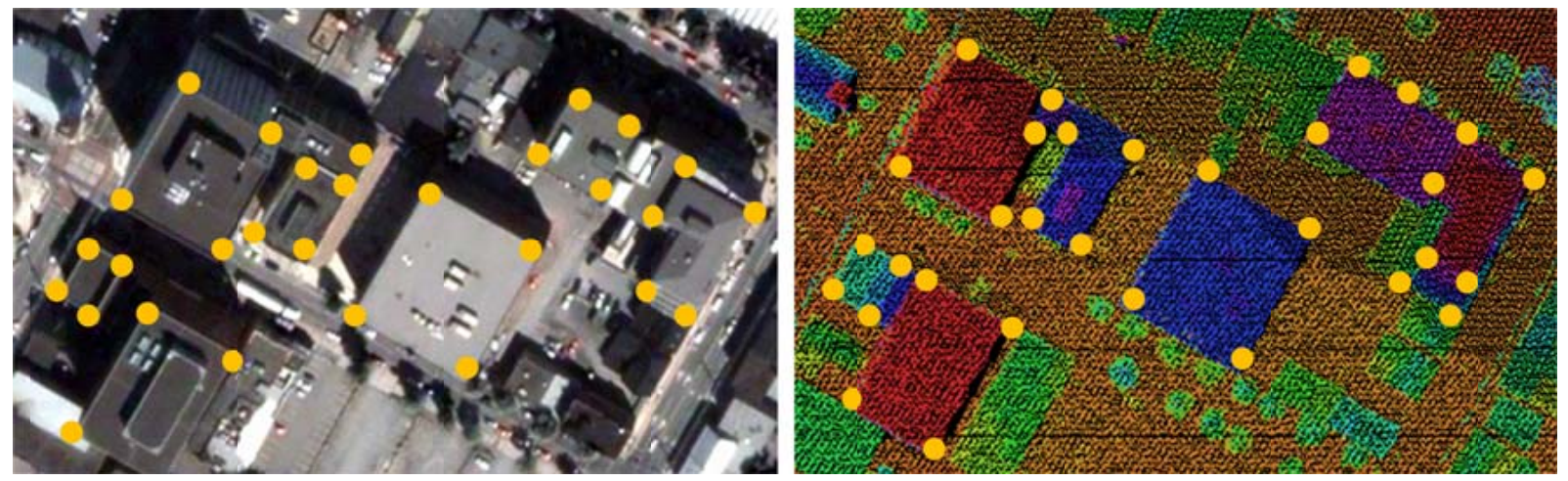

Fig. (1). Optical image (left) and corresponding LiDAR data (right). Notes: The yellow circles inthis Figure represent the building corners. These corners are the potential control points for optical image and LiDAR data registration.

The above steps are similar to steps requiredfor image-toimage registration [14]. Many successful methods have been developed for the registration of the optical images. However, given the substantially different imaging principles between optical and LiDAR sensors, the captured data sets exhibit substantially different characteristics. Therefore, the method proposed for image-to-image registration cannot be directly applied to register optical images and LiDAR data. Intensity based methods for image registrations are not suitable for optical images and LiDAR data registration since there are substantial differences in their data characteristics. The 3-D surface can be generated using photogrammetry techniques and then registration of optical images and LiDAR data can be done [15]; however, it requires overlapping optical images and processes are not automatic. Feature-based techniques are suitable for this type of registration as features can be extracted both from optical images and LiDAR data. The result of featured-based technique depends on the similarity of image features determined by feature extraction algorithms. The main problem with optical images and LiDAR data registration is to determine correct control points from both data sets and then their accurate matching. As discussed above, due to the importance of optical image and LiDAR data registration in many remote sensing applications, several methods have been developed over timeto solve the problems of this type of registration. These methods are categorized as per their types and discussed as follows in detail:

\subsection{Featured Based Registration}

Feature-based methods have been found better than the intensity based registration methods as manysimilar features are present in both optical images and LiDAR data sets. These methods depend on the features determined by feature extraction algorithms. The detection of control-points depends on feature extraction as control points that correspond to positions of image fragments with particular features [14]. Therefore, feature extraction is crucial for successful optical image and LiDAR data registration. Linear features, corners, lines and polygons are considered as features here.

\subsubsection{Corner}

Corners are the most common features used for image-toimage registration[16]. The advantages of using corner points are their uniqueness and high precision in their localization. Wong and Orchard [13] used modified Harris corner detector [17] to detect corners control points) from the optical image and then corresponding control points from the LiDAR data was extracted throughexhaustive search.

\subsubsection{Straight Lines}

An optical image and LiDAR data registration method proposed by Habib et al. [18] utilizes straight line features extracted from both data sets and 3-D similarity transformation for aligning the optical image relative to the corresponding LiDAR data reference frame. The straight line features are used due to the fact that the straight lines can be reliably, accurately and automatically extracted from both optical images and LiDAR data. In this approach, first, planer surfaces from LiDAR data are extracted and adjacent planes are intersected to determine 3-D straight line segments. Secondly, linear features from optical images are obtained through aerial triangulation. A mathematical model for expressing the necessary constraints for the alignment of conjugate optical imagesand LiDAR data straight lines is established. This model ensures that the corresponding straight lines are collinear after registering two data sets relative to a common reference frame.

Habib et al. [19] introduced another approach which starts by manipulating the photogrammetric imagery to produce a 3-D model, including a set of linear features along object space discontinuities, relative to an arbitrarily chosen coordinate system. Then, conjugate photogrammetric and LiDAR straight line features are used to establish the transformation between the arbitrarily chosen photogrammetric coordinate system and the LiDAR reference frame.

Habib et al. [20] proposed an alternative method for the purpose of integrating LiDAR data into photogrammetric triangulations. Two methodologiesare introduced that utilize straight line and aerial features derived from both data sets as primitives. The first methodology directly incorporates LiDAR lines as control information in the photogrammetric triangulation, while in the second methodology, LiDAR patches are used to geo-reference the photogrammetric model.

Deng et al. [21] proposed a registration procedure in which first straight lines are detected using Canny edge detection. Then pair-wise correspondence of detected straight lines is achieved by matching the straight-line pairs detected from LiDAR point clouds and optical images using 
generalized point photogrammetry. In the generalized point photogrammetry, all kinds of features are treated as generalized points and incorporated into extended collinearity equations [22]. The collinear condition is applied in generalized point photogrammetry to match straight lines.

\subsubsection{Sensor Invariant Features}

Schenk andCsatho [15] fused aerial images with LiDAR data to obtain better scene description of topography. This method utilizes the sensor invariant features found in both data sets at the registration stage.Such features correspond to the same object space phenomena, for example breaklines and surface patches. Matched sensor invariant features have been used to establish a common reference frame. Featurelevel fusion has been performed with sensor specific features that are related to surface characteristics.

\subsubsection{Building Roofs}

Building roofs are frequently used for optical images and LiDAR data registration as building roofs are linear features and available in both data sets. A method for optical images and LiDAR data registration has been proposed by Kwaket $a l$. ,[23] which uses centroids of plane roof surfaces as control information. This research was done on scenes where roofs are plane. The centroids of the plane roofs were extracted from the optical image using Canny edge detector. Then, Local Maximum Filter was used to extract centroids of the plane roofs fromthe LiDAR data. The extracted centroids from the LiDAR data were used as control information to compute exterior orientation parameters of optical (aerial) imagery. For verification purposes, exterior parameters were computed using GCPs(Ground Control Points) and the accuracy of registration is evaluated. This research shows that the centroid of a building roof is a useful source of control information.

Shorter and Kasparis [24] proposed a registration method which uses building roofs resent in optical image and LiDAR data. In this method, first, buildings present in an optical image and LiDAR data were detected. Then, the LiDAR data was interpolated to a fixed point spacing (DSM) to produce a binary building mask. In the binary building mask image, the bright pixels correspond to buildings and dark pixels to everything else. In the next step, buildings from the optical image were detected and then the binary building mask was generated. The 2-D Fourier transform and 2-D log polar Fourier transform of both images werecomputed. The computed 2-D Fourier transforms and phases of the log polar 2-D Fourier transforms of the optical image and LiDAR data were used to compute parameters for translation, rotation and scaling geometric transformations [25]. The computed transformation parameters werethen used to register optical imagesandLiDAR data.

\subsubsection{Displacement Correction Method}

A two-step displacement correction method for LiDAR point cloud and aerial images registration is proposed by $\mathrm{Wu}$ et al. [26].This is a linear registration approach for LiDAR data and aerial images registration without using orientation parameters. Pre-processing is performed on LiDAR data to classify the point cloud into ground-points, building points, and above ground points. These classified LiDAR point clouds are used in two displacement correction steps to select control points for the registration.These two displacements corrections are tilt displacement corrections and height displacement corrections. The tilt displacement is defined as displacement caused by the non-parallelity of the image plane and the datum plane in the object space. An algorithm has been developed to remove tilt displacement which creates a functional relationship to map the datum plane to the image plane. The height displacement correction algorithm has been developed to remove the displacement caused by the perspective geometry of the camera and the height difference of each LiDAR point relative to the datum plane. The control points are selected manually in the LiDAR point cloud and aerial image respectively. The control points are divided into two groups; one group is ground points which are used for tilt displacement correction. Another type of control points are object points. Object points are mainly roof corners, which are used for height displacement correction.

\subsubsection{Summary}

As per the above discussions, it has been investigated that the feature-based registration method is relatively well suited for optical images and LiDAR data as these data sets contains enough distinctive and easily detectable objects required for registration. Registration of optical images and LiDAR data has been often performed by using building roofs as control information; however, the use of roof as control information is limited by the fact that many natural scenes do not contain buildings. Mostly remote sensing data contains both manmade objects such as buildings, roads, etc. and natural objectssuch as rivers, forests, etc. The featurebased registration methods are not veryeffective for the natural settings. Furthermore, the main problem with feature based registration is that the optical image and LIDAR data often capture different feature characteristics that make feature-based techniques less effective.A two-step displacement correction algorithm for optical image and LiDAR data registration without using orientation parameters proposed by $\mathrm{Wu}$ et al. [26] have achieved the same accuracy level as the traditional photogrammetric space resection algorithm. However, the problem associated with this approach is that the selections of control points are manual.The advantages and limitations of feature based algorithms are discussed in Table $\mathbf{1 .}$

\subsection{Mutual Information Based Registration}

As discussed in aforesaid registration methods, the majority of feature based registration methods utilizes only one type of features. However, as characteristics of optical images and LiDAR data are very different and many features available in one data set may not be available in others, registration using single features does not produce accurate registration results. To overcome this problem, statistical and information-theoretic methods have been extensively used for multi-modal image registration. This method is called mutual information(MI) registration, which provides a means to measure statistical dependence between two random variables or the amount of information that one variable contains about other. The basic concept behind the use of MI for registering images is that MI (similarity matrix) calculated from the two images will reach its maximum MI when the images are perfectly geometrically 
Table 1. Advantages and Limitations of Feature-Based Algorithms

\begin{tabular}{|c|c|c|}
\hline Category & Advantages & Limitations \\
\hline Corners & $\begin{array}{ll}\text { - } & \text { Uniqueness and high precision in their localization } \\
\text { - } & \text { Suitable for urban environment } \\
\text { - } & \text { Fast computation }\end{array}$ & $\begin{array}{l}\text { - } \quad \text { Same corners may not be present in optical images and } \\
\text { LiDAR data } \\
\text { - } \quad \text { Not suitable for natural environment }\end{array}$ \\
\hline Straight lines & $\begin{array}{l}\text { - Can be reliably, accurately and automatically extracted } \\
\text { from both optical image and LiDAR data } \\
\text { - Suitable for urban environment }\end{array}$ & $\begin{array}{l}\text { - } \quad \text { Need overlapping optical images to generated 3-D model } \\
\text { - } \quad \text { Not suitable for natural environment }\end{array}$ \\
\hline Sensor invariant features & $\begin{array}{l}\text { - } \begin{array}{l}\text { Breaklines and surface patches are common in optical } \\
\text { images and LiDAR data }\end{array} \\
\text { - } \quad \text { Suitable for both urban and natural environment }\end{array}$ & $\begin{array}{l}\text { - } \quad \text { Need stereopair aerial images to orient aerial imageswith } \\
\text { LiDARdata } \\
\text { - } \quad \text { Computationally expensive }\end{array}$ \\
\hline Building roofs & $\begin{array}{l}\text { - } \quad \text { Suitable for urban environment } \\
\text { - } \quad \text { Excellent source of information for control points }\end{array}$ & $\begin{array}{l}\text { - } \quad \text { Same building structures may not be present in optical } \\
\text { images and LiDAR data } \\
\text { - } \quad \text { Performance is poor if building roofs are complex } \\
\text { - } \quad \text { Not suitable for natural environment }\end{array}$ \\
\hline $\begin{array}{l}\text { Displacement correction } \\
\text { method }\end{array}$ & $\begin{array}{l}\text { - } \quad \text { Better registration accuracy } \\
\text { - } \quad \text { There is no need of orientation parameters }\end{array}$ & $\begin{array}{l}\text { - } \quad \begin{array}{l}\text { Manual selection of control points from optical images } \\
\text { and LiDAR data }\end{array} \\
\text { - } \quad \text { Not suitable for natural environment }\end{array}$ \\
\hline
\end{tabular}

Table 2. Advantages and Limitations of Optical Images and LiDAR Data Registration Methods

\begin{tabular}{|c|c|c|}
\hline Category & Advantages & Limitations \\
\hline Feature-based methods & 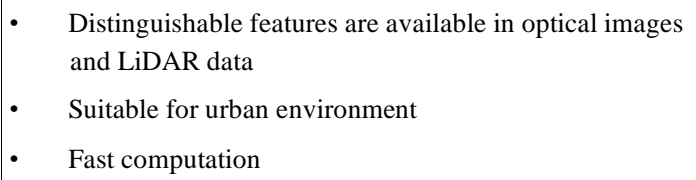 & $\begin{array}{l}\text { - Optical images and LIDAR data often capture different } \\
\text { feature characteristics } \\
\text { Not suitable for natural environment }\end{array}$ \\
\hline $\begin{array}{l}\text { Mutual information based } \\
\text { methods }\end{array}$ & $\begin{array}{l}\text { - } \quad \text { It includes different features at the same time } \\
\text { - } \quad \text { Suitable for urban environment }\end{array}$ & - Not suitable for natural environment \\
\hline Frequency-based methods & - $\quad$ Fast computation & $\begin{array}{l}\text { - } \quad \text { Uses only LiDAR intensity images } \\
\text { - } \quad \text { Low registration accuracy }\end{array}$ \\
\hline $\begin{array}{l}\text { Salient point based } \\
\text { methods }\end{array}$ & $\begin{array}{ll}\text { - } & \text { Suitable for urban and natural environment } \\
\text { - } & \text { Better registration accuracy }\end{array}$ & $\begin{array}{l}\text { - } \quad \text { Computationally expensive } \\
\text { - Do not consider relief displacement present in HR } \\
\text { imagery }\end{array}$ \\
\hline
\end{tabular}

aligned [27]. A MI based approach to register optical images with LiDAR data has also been proposed by Mastin et al, [8]. This approach evaluates registration statistics in the 2-D image plane via projection of LiDAR features within the constraints of a camera model for comparison with the image features. MI based registration methods seek the camera matrix that maximizes the MI between the distribution of image features and projected LiDAR features.

MI is defined in terms of entropies of the optical image features, LiDAR data features and their joint entropy[8]:

$I\left(u, v_{r}\right)=H(u)+H\left(v_{r}\right)-H\left(u, v_{r}\right)$

where $\mathrm{u}$ is the features in an optical image and $\mathrm{v}_{\mathrm{T}}$ is the features in projected LiDAR data in 2-D space.

The entropy of optical image features remains constant and the entropy of the LiDAR features remains approximately constant. The registration algorithm discussed in [8] renders 3-D LiDAR data points onto the optical image plane for evaluating statistics. The three different methods for evaluating MI between optical image and projected LiDAR data have been proposed. The first method simply uses the mutual information between elevation in the LiDAR point cloud and luminance in the optical image. The point cloud is rendered with height intensities, where the brighter point indicates a higher elevation. Only image pixels that have corresponding projected LiDAR points are used for calculating registration statistics. The reason for doing this is that the visual appearance of urban scenes tends to vary structurally by height for architectural regions. Also, there is measurable dependence between the optical appearance and the measured LiDAR height. A scene shown by both modalities is shown in Fig. (2a) and Fig. (2b). The second method uses the mutual information between luminance in 


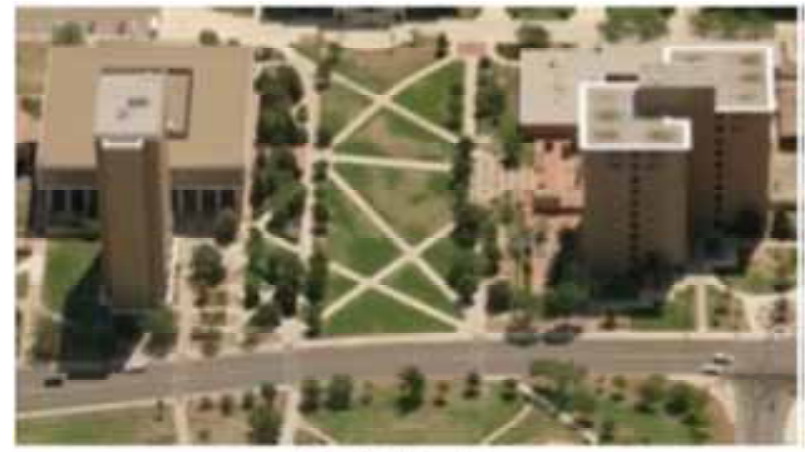

(a) Optical image

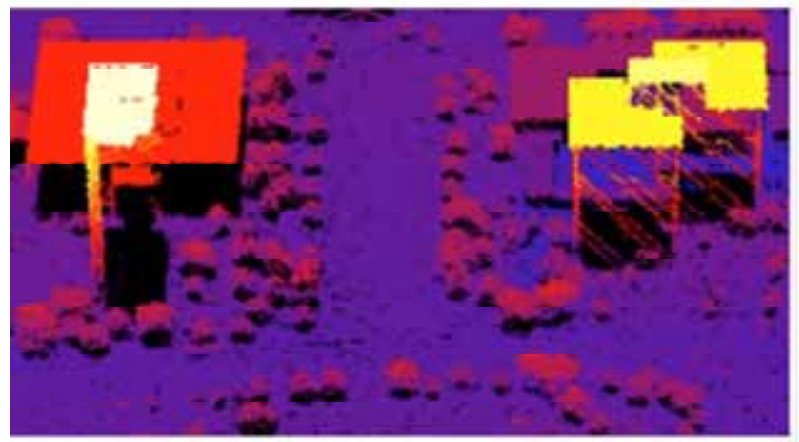

(b) Height encoded LiDAR image

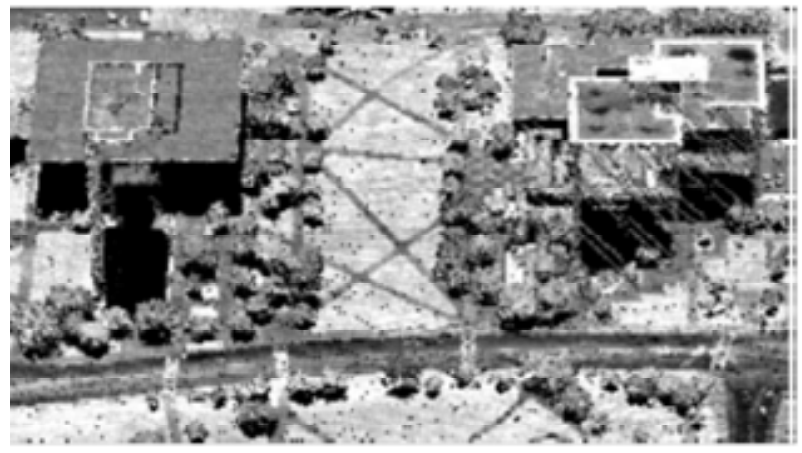

(c) LiDAR intensity image

Fig. (2). Detail of optical and LiDAR data. (a) Shows an optical image of two buildings with pathways in between, (b) shows the registered LiDAR data set of the same scene with intensity encoded, while (c) shows the LiDAR data with the Pdet attribute. Courtesy: (Mastin et al. 2009), Computer Vision and Pattern Recognition, CVPR 200, IEEE Conference.

the optical image and intensity values in the LiDAR point cloud. A LiDAR intensity image is shown in Fig. (2c). The walkway between the buildings can easily be seen in the LiDAR intensity image. Finally, the third method calculates entropy among optical image luminance, LiDAR elevation and LiDARintensity values. It is assumed that the LiDAR intensity image is statisticallyindependent of the LiDAR elevation image. This leads to the following joint entropy:

$$
H\left(u, v_{e}, v_{i}\right)=H\left(u, v_{e}\right)+H\left(u, v_{i}\right)
$$

where $u$ is the image luminance, $v_{\mathrm{e}}$ is the LiDAR elevation, and $v_{i}$ is the LiDAR intensity values.Based on the joint entropy the registration statistics is calculated. The oblique aerial photographs are used in this research since this research is aimed to produce 3-D models of urban scenes.

\subsubsection{Summary}

From the MI based registration methods, it can be concluded that the mutual information based methods are better than the feature based methods as they include different features at the same time. The mutual information based registration method proposed by Mastinet al. [8] has been achieved using 3D-2D rendering of height and probability of detection attributes of theLiDAR system. The registration accuracy is shown on average to be $90 \%$ when both LiDAR elevation and a LiDAR intensity images are used in registration. The registration accuracy is slightly less when only LiDAR elevation image is used which shows that the use of a LiDAR intensity image can improve registration accuracy. The mutual based registration methods have shown their effectiveness for urban scenes; however, it is ineffective for natural scenes as information-theoretic methods cannot be applied to natural scenes.

\subsection{Frequency Based Registration}

Frequency based methods have been frequently used in optical image registration $[25,28,29]$. Frequency based methods use phase to determine the alignment between two images. One of the popular frequency based method is phase correlation, which has been extended to handle geometric distortions such as rotation and scaling [25] These frequency based methods cannot be applied directly to register optical images and LiDAR data due to differences in data characteristics. An improved version of the frequency based method to register optical images and LiDAR data has been developed byWong and Orchard [13]. In this proposed algorithmthe control points are extracted only from an optical image. To extract control points, this algorithm applies local normalization on an optical image to compensate for non-uniformity in image illumination and contrast. Then a modified Harris corner detector is applied [17]. Only the strongest control points are selected based on threshold values. Once control points from optical image are detected, an exhaustive region correspondence search algorithm is applied to find the optimal correspondence between regions from an optical image to a LiDARimage. Fourier transform (FFT) [30] has been used to reduce the cost of an exhaustive search. The problem of different intensity image characteristics of optical and LiDAR images have been solved through an integrated local intensity mapping transformation optimization process.

Wong and Orchard [13] used the 4th random sample consensus (RANSAC) algorithm to remove incorrect control-point pairs to improve registration process. A final set of control points in the optical and the LiDAR intensity image are shown in Fig. (3). The method proposed by Wong and Orchard [13] determines the image transformation model and estimates the transformation parameters from the initial sample of control-points. This method calculates close values of transformation parameters (e.g. sift, rotation, and scale) for the corresponding control-points.

\subsubsection{Summary}

The frequency based method used for optical image and LiDAR data registration uses only LiDARintensity imageswhich contain less information about the topography as compared to LiDAR elevation images. A LiDAR intensity 


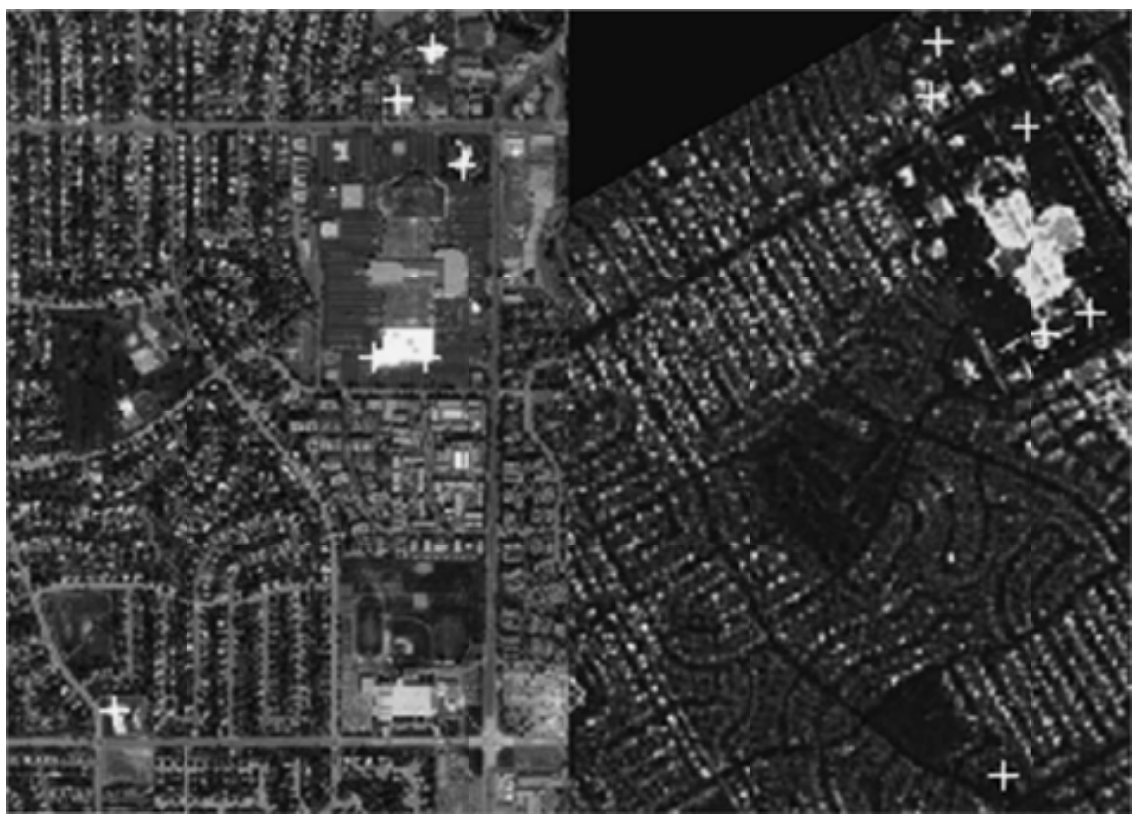

Fig. (3). Final set of detected control points (as indicated by crosses). (Left) Optical image. (Right) LiDAR image.Note: This Figure shows the effectiveness of the methodology developed by Wong and Orchard [13] in control point detection. Courtesy: (Wong and Orchard 2008), IEEE transactions on Geoscience and Remote Sensing.

image can be used to improve registration accuracy; however, using only LiDAR intensity images for registration purposes is not enough to produce accurate registration results. The registration method produced by Wong and Orchard [13] has introduced the region correspondence search that usesFourier transform (FFT) [30] to reduce the cost of an exhaustive search. Wong and Orchard [13] claims that the developed methodology for optical images and LiDAR data registration produces good level of accuracy under various difficult optical and LiDAR image pairs. Wong and Orchard [13] have used orthorectified low resolution optical images to test the developed algorithms; however, today very high resolution satellite imagery is available which is more useful for various remote sensing applications.

\subsection{Salient-point Based Registration}

The optical image and LiDAR data registration techniques discussed in the above section are mostly suitable for man-made settlements and are not well suitable for the natural scenes where building, roads, etc. are not present. Considering the general type of features present in remote sensing data a salient point approach with combination of multiscale image analysis [16] is helpful to improve overall registration accuracy. The general overview of a salientpoint detector in the context of image matching is given in $[31,32]$. Salient points in images refer to image locations with distinctive features providing discrimination between objects of interest and the background [33]. A salient-point detector is a visual operator used to find image locations or regions containing object of interests [34-39]. A salient-point visual operator can detect various types of salient features such as corners, lines, junctions, intensity blobs, and homogenous regions $[16,35,40,41]$. Although, these techniques have proven their effectiveness for control-point extraction, there are, however, many weaknesses [33]. Feature extraction and salient point detection using these techniques gives poor results at a large scale during image analysis. Irrelevant sharp edges may become more highlighted than salient regions and corners [32, 34, 38]. Difficulties in salient-points detection appear when edges in images are not sharp or corrupted by noise.

To overcome the aforesaid shortcomings [33] proposeda new method of control point extraction using a salient visual operator. The purposed solution was designed with the intention to registeroptical images and LiDARdata automatically with high accuracy.The development of control point extraction schemes starts with an extraction of image fragments which satisfy given requirements for intensity and shape features(local features). The centers of these image fragments wereconsidered as control points. The control point extraction wasrealized using the concept of multiscale salient image discs (SIDs). The multiscale SID concept is anextension of a salient point method of feature extraction formultiscalehigh-contrast homogenous regions $[34,35]$. "A SID is defined [33] as a circular image fragment of a variable diameter, which is inscribed into a homogeneous region (i.e., is tangent to the background in at least two points) and has the local maximum of contrast-tohomogeneity ratio". Three SID image descriptors were introduced: 1) planar pose characteristics (e.g. center coordinates, local scale, and local orientation); 2) planar shape descriptors; and 3) image intensity descriptors. The control points selected are the centers of those SIDs .

In the approach of Palenichka and Zaremba [33], amultiscale isotropic matched filtering (MIMF) was developed to effectively identify candidate SID positions. MIMFwasused to extract SIDs from images in a way that is invariant to translation, scale and rotation. The MIMF operator was originally developed by Palenichka and Zaremba [41] to detect individual trees in LiDAR images. This MIMF operator was modified to detect SIDs both from optical images and LiDAR data. This operator does not 


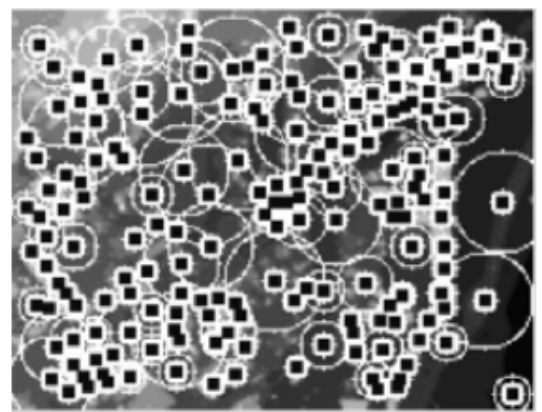

(a)

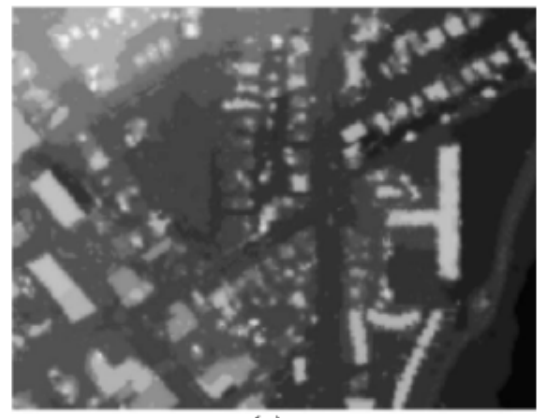

(c)

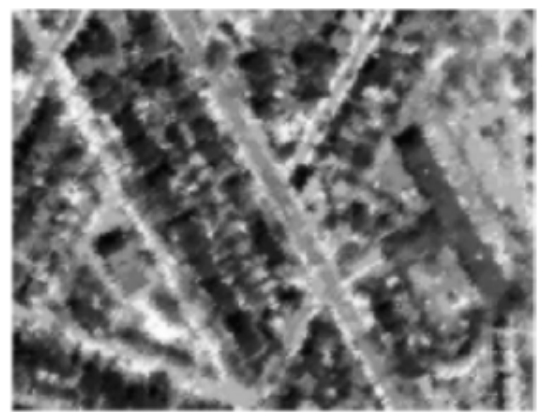

(b)

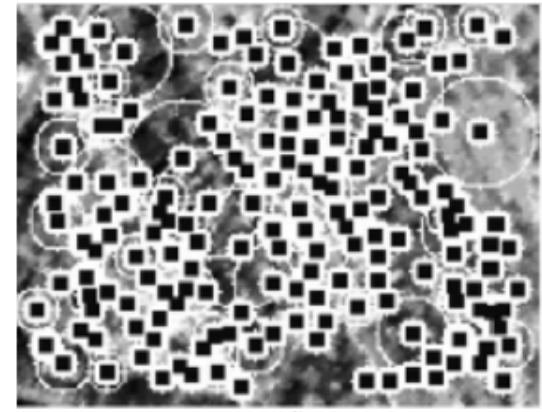

(d)

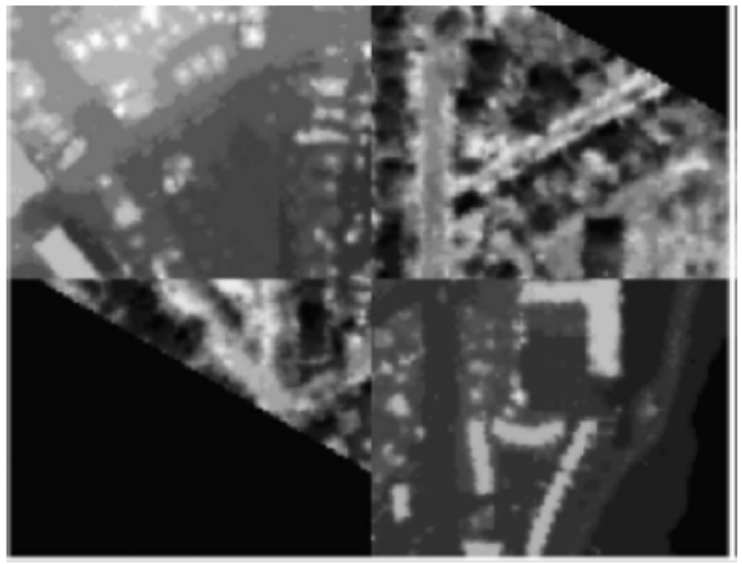

(e)

Fig. (4). Example of a heterogeneous image registration (Ottawa test area). (a) A LiDAR height image. (b) A rotated and shifted panchromatic image. (c) SID extraction from a LiDAR image. (d) SID extraction from a panchromatic image. (e) An image registration result. Courtesy: (Palenichka and Zaremba 2010) IEEE transactions on Geosciences and Remote Sensing.

require pre-segmentation images. The MIMF operator produces two sets of detected control points from optical images and LiDAR data.

Intersection matching distance (IMD) algorithm was developed to establish pair wise correspondence between two sets of extracted SIDs. This algorithm is based on the minimization of a dissimilarity measures (i.e. a distance between two sets of descriptor vectors) over all possible subsets of control point SIDs detected from optical image and LiDAR data.

Experiments of the developed algorithms were done using Quickbird imagery and LiDAR generated DSM images. Optical images and LiDAR data for the forest and urban areas were registered to show the effectiveness of the developed algorithm. The performance of the SID extraction is evaluated by the receiver operating characteristics (ROC) method. The similarity transformation mapping method was used to estimate transformation models using SIDs pair correspondence. Fig. (4) shows the registration result with four quadrants: two diagonal quadrants from the LiDAR image and other two from the transformed optical image.

\subsubsection{Summary}

The multiscale version of a salient point approach is suitable when combining optical images with LiDAR data as both data sets may have different resolution. This technique facilitates automatic selection of multiple control points even for images of natural scenes. Moreover, multitude of control points with their relative location enhances the accuracy of registration. Location saliency ensures the uniqueness of control points in images which is important for control point extraction. The old saliency based methods have problems:a lack of explicit saliency conditions, poor results on feature extraction, and a high computational cost. The saliency based approach proposed by Palenichka and Zaremba [33] to 
register optical images with LiDARdata aims to overcome these problems of saliency based methods. This approach automatically extracts control points from optical images and LiDAR datausing transformation-invariant detection of image discs (SIDs). Pair-wise correspondence between two sets of extracted SIDs is determined by the calculation of the IMD. Finally registration of optical images and LiDAR data is performed using a similarity transformation model. This technique has shown good accuracy in control point extraction from both optical images and LiDAR data. Also, the registration results are relatively good particularly for natural scenes. However, the problem of relief displacement, which is apparently present in HR imagery, has not been addressed. Moreover, the developed technique is complex and iscomputationally expensive thus difficult to implement for real world applications.

\subsection{Analysis of the Advantages and Limitations of Different Approaches}

Many research works have been done on accurate registration of optical images with LiDAR data since integration of these two data sets is important for various remote sensing applications. In conclusion advantages and disadvantages of existing methods to register optical images with LiDAR data are listed in the Table $\mathbf{2}$ and discussed as follows:

\subsubsection{Feature-Based Methods}

Feature-based methods have been considered effective for the alignment of optical images with LiDAR data as optical and LiDAR sensors capture distinguishable features. Feature-based methods have been widely used for image-toimage registration. The feature-based methods have been modified to register optical images andLiDAR data. However, the main problem with feature based registration is that the optical images and LIDAR data often capture different feature characteristics that make feature-based techniques less effective. Also, the majority of feature based registration methods utilizes only one type of features. Furthermore, mostly remote sensing data contains both manmade and natural objects such as buildings, roads, and rivers, forests respectively. The feature-based methods are lesseffective for the regions where natural objects (river, lakes, forest, etc.) are present.

\subsubsection{Mutual Information (MI) Based Methods}

The methodology developed by Mastin et al. [8] to register optical images and LiDAR data shows that the MIbased methods are better compared to the feature-based methods as it includes different features at the same time. The registration accuracy was shown on average to be $90 \%$ when both LiDAR elevation and LiDAR intensity images are used in registration. The mutual based registration has shown its effectiveness for the urban scenes, however, it is ineffective for the natural scenes as information-theoretic methods cannot be applied to natural scenes.

\subsubsection{Frequency-Based Methods}

Most of the frequency based method used for optical images and LiDAR data registration uses only intensity images of LiDAR which contain less information about the topography compared to LiDAR elevation images. Also, the nature of optical images and LiDAR intensity images are very different in nature. The LiDAR intensity images can be used to improve registration accuracy; however, using only LiDAR intensity images for registration purpose is not enough to produce accurate registration result.

\subsubsection{Salient Point Based Methods}

A salient point approach with a combination of multiscale image analysis suggested by Palenichka and Zaremba [33] has shown relatively better result than the earlier registration techniques particularly for natural scenes. However, the problem of relief displacement present in HR optical images has not been addressed. Moreover, this technique includes complex algorithms which are computationally expensive and hence difficult to implement for real world applications.

\section{CONCLUSIONS}

Optical images and LiDAR data registration is a prerequisite to utilize the complementary characteristics of both data sets. Accurate registration between optical image and LiDAR data is vital for many remote sensing applications such as;feature extraction, image classification, $3 \mathrm{D}$ city modelling, and canopy modelling. This paper did a survey of the classical and up-to-date registration methods developed to register optical images and LiDAR data. The registration methods are grouped into different categories according to the nature of their calculation principles. Although many methods have been developed in the last decade for the registration of optical image and LiDAR data, however:

- In spite of different characteristics of optical images and LiDAR data, many methods are still using the previously developed algorithms for image-to-image registration.

- Automatic and accurate registration of these data sets still remains an open problem.

- No techniqueshave been found that can register optical images and LiDAR data automatically with the required accuracy and efficiency for practical applications

\section{FUTURE OUTLOOK}

As per the review of the existing registration methods, it has been found that the use of a multiscale saliency based approach is best suited to detect control points from both data sets. The interest point matching algorithm [42], which provide accurate and efficient matching of image control points in optical images may have potential to be extended to match detected control points from optical images and LiDAR data. The problem of relief displacement of HRimagery is another issue for accurate registration between optical image and LiDAR data. It is also important to incorporate sensor information of optical and LiDAR sensors to solve the relief displacement problem.

To achieve the goal of automatic and accurate registration of optical images and LiDAR data, it is worth to explore the appropriate algorithms from existing registration methods and developnew algorithms. 


\section{CONFLICT OF INTEREST}

The authors confirm that this article content has no conflicts of interest.

\section{ACKNOWLEDGEMENT}

This research was funded by the Canada Research Chairs Program.

\section{REFERENCES}

[1] Kim C, Ghanma M, Habib A. Integration of Photogrammetric and LIDAR data for realistic 3D model generation. 2006, [Online]. Available from: http://regard.crg.ulaval.ca/2006/proceedings/13kim_et_al.pdf [Accessed 27 October 2011].

[2] Baltsavias E. A comparison between photogrammetry and laser scanning. ISPRS J Photogramm Remote Sens 1999; 54(1): 83-94.

[3] Rottensteiner F, Jansa J. Automatic extraction of building from LIDAR data and aerial images.In: Proc. Internat. ISPRS 2002; 34(4): 295-301.

[4] LiHaitao, Haiyan GU, Jinghui Y. Fusion of High-Resolution Aerial Imagery and LiDAR data for Object-oriented Urban Land-Cover Classification, ISPRS Workshop on Updating Geo-spatial Databases with Imagery \& The 5the ISPRS Workshop on DMGISs 2007; Urumchi, Xingjiang, China.

[5] Cui LL, Tang P, Zhao ZM. Study on object-oriented classification method by integrating various features. J Remote Sens 2006;10(1); 104-10.

[6] Syed S, Dare P, Jones S. Automatic classification of land cover features with high resolution imagery and lidar data: an objectoriented approach. Proceedings of SSC 2005 Spatial Intelligence, Innovation and Praxis: The national biennial Conference of the Spatial Sciences Institute Sept 2005.

[7] Park JY, Shrestha RL, Carter WE, Tuell GH. Land-cover classification using combined ALSM (LIDAR) and color digital photography. Presented at ASPRS conference, St. Louis, Missouri, April 2001; pp 23-27.

[8] Mastin A, Kepner J, Fisher J. Automatic registration of LIDAR and optical images of urban scenes. Comput Vis Pattern Recognit 2009; 2639-46.

[9] Brenner C. Building reconstruction from images and laser scanning. Int J Appl Earth Obs 2005; 6(3-4): 187-98.

[10] Chen L, Chiang T, Teo T. Fusion of LIDAR data and high resolution images for forest canopy modelling. In: Proc 26th Asian Conf on Remote Sensing 2005.

[11] Habib A, Schenk T. New approach for matching surfaces from laser scanners and optical sensors. Int Arch Photogramm Remote Sens1999; 32(3W14): 55-61.

[12] Postolov Y, Krupnik A, McIntosh K. Registration of airborne laser data to surfaces generated by photogrammetric means. Int Arch Photogramm Remote Sens 1999; 32(3W14): 5-99.

[13] Wong A, Orchard J. Efficient FFT-Accelerated Approach to Invariant Optical-LIDAR Registration. IEEE T Geosci Remote 2008; 46(11): 3917-25.

[14] Zitova B, Flusser J. Image registration methods: a survey. Image Vis Comput 2003; 21(11): 977-1000.

[15] Shenk T, Castho, B. Fusion of LiDAR data and aerial imagery for a more complete surface description. ISPRS Int Arch 2002; 34(3A): 310-31.

[16] Zheng Z, Wang H, Teoh EK. Analysis of gray level corner detection. Pattern Recogn Lett 1999; 20(2): 149-62.

[17] Noble A. Descriptions of image surfaces. Ph.D. dissertation, Oxford, UK:Oxford University 1989.

[18] Habib A, Ghanma MS, Morgan MF, Mitishita EA. Integration of laser and photogrammetric data for calibration purposes. International Archives of XXth ISPRS Congress, July 12-23 Commission I 2004 Istambul, Turkey.
[19] Habib A, Ghanma M. Morgan M, Al-Ruzouq R. Photogrammetric and LiDAR data registration using linear features. Photogramm Eng Remote Sens 2005; 71(6): 699-707.

[20] Habib A, Shin S, Kim C, Al-Durgham M. Integration of photogrammetric and LiDAR data in a multi-primitive triangulation environment. Innovations in 3D Geo Information Systems; Berlin: Springer 2006. Part 2, pp.29-45.

[21] Deng F, Hu M, Guan H. Automatic registration between LiDAR and digital images, Int Arch photogramm Remote Sens Spatial Inf Sci 2008; 37(B1): 487-90.

[22] Zhang Z, Zhang Y, Zhang J, Zhang H. Photogrammetric modeling of linear features with generalized point photogrammetry. Photogramm Eng Remote Sens 2008; 74(9): 1119-27.

[23] Kwak TS, Kim Y, Yu KY, Lee BK. Registration of aerial imagery and aerial LiDAR data using centroids of plane roof surfaces as control information. KSCE J Civ Eng 2006; 10(5): 365-70.

[24] Shorter N, Kasparis T. Autonomous registration of lidar data to single aerial image. geoscience and remote sensing symposium. IGARSS 2008. IEEE Int 2008; 5: 216-19.

[25] Reddey BS. Chatterji BN. An FFT-Based technique for translation, rotation, and scale-invariant image registration. IEEE Trans Image Process 1996; 5(8): 1266-71.

[26] Wu H, Li Yong, Jonathan Li, Gong J. A Two-step displacement correction algorithm for registration of lidar point clouds and aerial images without orientation parameters. Photogramm Eng Remote Sens 2010; 76(10): 1135-45

[27] Viola P, Wells W. Alignments by maximization of mutual information. Int J Comput Vision 1997; 24(2): 137-54

[28] Castro E, Morandi C. Registration of translated and rotated images using finite Fourier transforms. IEEE Trans Pattern Anal Mach Intell 1987; 9(5): 700-3.

[29] Zavorin I, Moigne JL. Use of multiresolution wavelet feature pyramids for automatic registration of multisensor imagery. IEEE Trans Image Process 2005; 14(6): 770-82.

[30] Cooley J, Tukey J. An algorithm for the machine calculation of complex Fourier series. Math Comp 1965; 19(90): 297-301.

[31] Sebe N, Lew MS. Comparing salient point detectors. Pattern Recog Lett 2003; 24(1-3): 89-96.

[32] Lindeberg T. Feature detection with automatic scale selection. Int J Comput Vision 1998; 30(2): 77-116.

[33] Palenichka RM, Zaremba MB. Automatic extraction of control points for the registration of optical satellite and LiDAR images. IEEE Trans Geosci Remote Sens 2010; 48(7): 2864-79.

[34] Kadir P, Brady M. Saliency: scale and image description. Int J Comput Vision 2001; 45(2): 83-105.

[35] Itti L, Koch C, Niebur E. Amodel of saliency-based visual attention for rapid scene analysis. IEEE Trans Pattern Anal 1998; 20(11):1254-59.

[36] Reisfeld D, Wolfson H, Yeshurun Y. Context-free attentional operators: The generalized symmetry transform. Int J Comput Vis 1995; 14(2): 119-30.

[37] Tagare HD, Toyama K, Wang JG. A maximum-likelihood strategy for directing attention during visual search. IEEE Trans Pattern Anal 2001; 23(5): 490-500.

[38] Lowe DG. Distinctive image features from scale-invariant keypoints. Int J Comput Vis 2004; 60(2): 91-110.

[39] Harris C, Stephens M. A combined corner and edge detector. In: Proc $4^{\text {th }}$ Alvey Vis Conf. (AVC88). Univ. Manchester 1988. pp. 147-51.

[40] Alhichri HS, Kamel M. Virtual circles: a new set of features for fast image registration. Pattern Recogn Lett 2003; 24(9-10):1181-90.

[41] Palenichka RM, Zaremba MB. Object shape extraction based on the piecewise linear skeletal representation. In: ICIAR'05 Proceedings of the second international conference on image analysis and recognition. IEEE Toronto section. Heidelberg: Springer-verlag Berlin 2005.

[42] Xiong Z, Zhang Y. A critical review of image registration methods. Int J Image Data Fusion 2010; 1: 137-58. 